\title{
Explore the Mystery of the Origin of the Double Helix and the Biomembrane
}

\author{
Xiangchen Yin \\ Key Laboratory of Inorganic Chemistry in Universities of Shandong Province, Department of Chemistry and \\ Chemical Engineering, Jining University, Qufu, Shandong, China \\ Email: xcy561029@sina.com
}

Received November 2014

\begin{abstract}
In nature, the most possible reason that helix is chosen as the basic structure of life molecule is based on its simplest chiral three-dimensional structure. The process of the conversion from chemical molecules to double helical molecules is completed by the topology effect which belongs to the simplest way to form helix, and no external power is needed; moreover, the energy of double helix has fixed drive direction [1]. The dual-branch loop helix (II)-the transition state of double helix has many uses, for example, it can be turned to double helix, and it may be broken into two fragments of $\alpha$ and $\beta$ which can construct more complicated structures. So the dualbranch loop helix (II) can provide special "building block" of assembling biomembrane and other life molecules.
\end{abstract}

\section{Keywords}

Topology, Double Helix, Dual-Branch Loop Helix, Building Block, Biomembrane

\section{Formation of the Double Helix}

It is not difficult to form chain molecules from small molecules, ring molecules from chain molecules, rotaxane from ring molecules or chain molecules, and catenane from rotaxane. As exhibited in Figure 1, a ring molecule can be built by a chain molecule, and a double linked ring can be constructed by one chain and one ring or a rotaxane, in addition, a tetraplex ring (I) can be made from two double linked rings or two rotaxanes.

As shown in Figure 2, it has been proved that a tetraplex ring I and a dual-branch loop helix II are of topological equivalence in mathematics [2]; dual-branch loop helix II and double helical loop III are of topological equivalence, so there is no doubt that I, II and III are of mutually topological equivalence.

Obviously, III is the closure of the double helix, which can form a super helix, and the super helix just represents the DNA. It is clear that dual-branch loop helix II gives birth to the double helix III. The surrounded number of dual-branch loop helix II can be an arbitrary integer, so a double helix of any length can be formed.

It is more exciting that the dual-branch loop helix II can be broken into two fragments of $\alpha$ and $\beta$ which can be used as "building blocks" to assemble into more basic, more complex and more important living matter. The above fracture modes have been illustrated in Figure 3. 

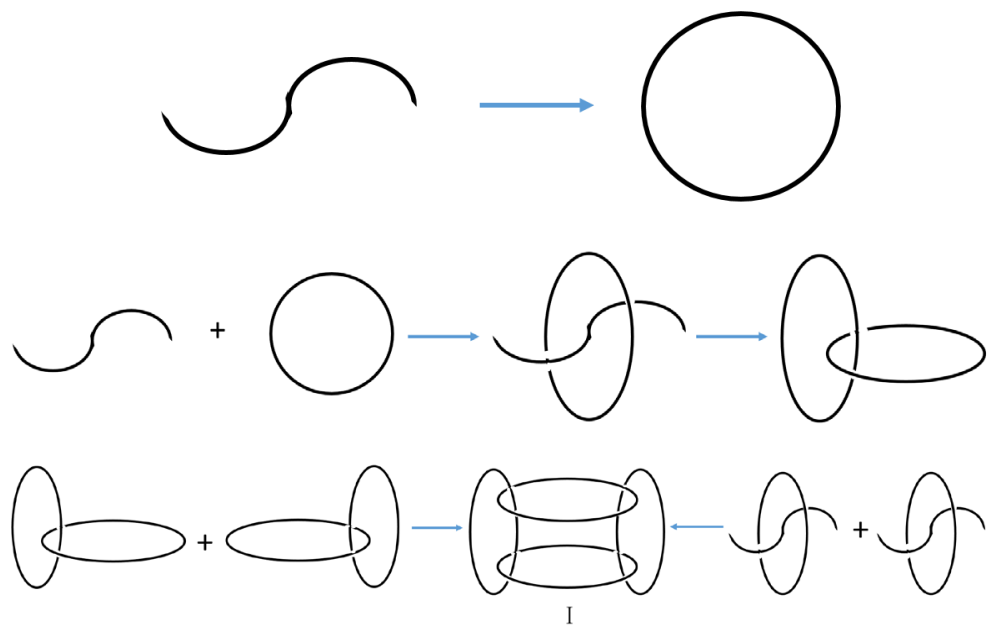

Figure 1. Formation of the tetraplex ring I.

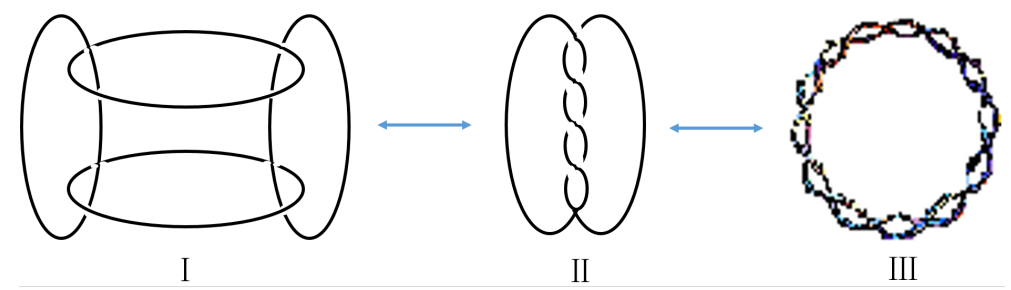

Figure 2. The topological equivalence of I, II and III.

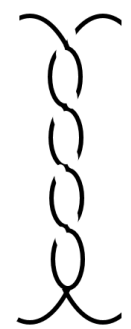

$\boldsymbol{\alpha}$

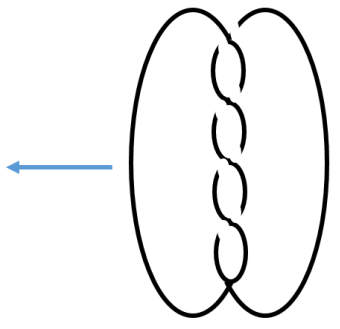

II

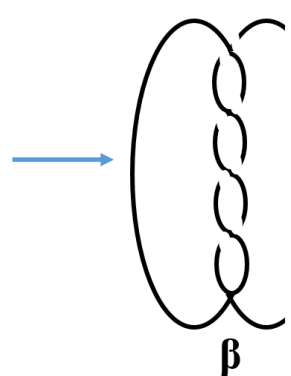

Figure 3. Fracture modes of the dual-branch loop helix II.

\section{Assembly of "Building Blocks"}

1) Longer helical chains are formed when several or more block $\alpha$ are assembled vertically. The helical chains that are long enough can form trefoil knots.

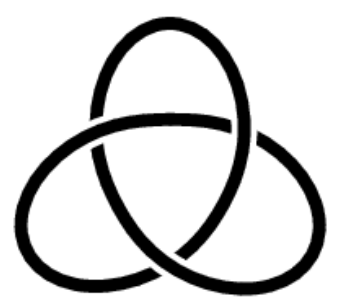

2) Two "building blocks" can connect separately with each other shoulder to shoulder in parallel to form a net belt, or two "building blocks" can horizontally connect with each other to form a net belt. Net belts can take a variety of combinations: 
a) Net belt assembled to form a trefoil knot

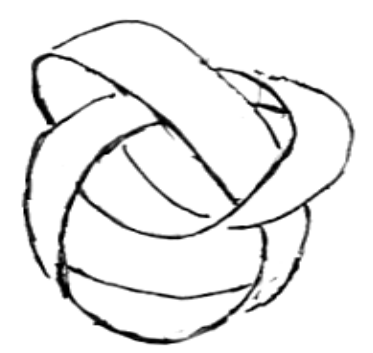

b) Net belts assembled to form a Mobius strip [3]

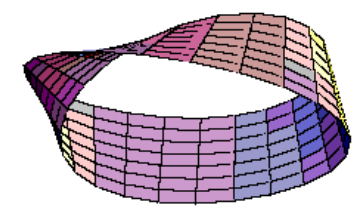

c) Net belts assembled to form a helical ball

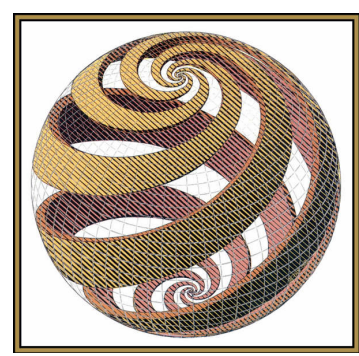

d) Net belts assembled to form a "helical braid"

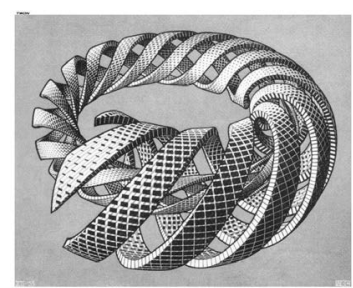

3) Two "building blocks" vertically and horizontally assembled to form a square network

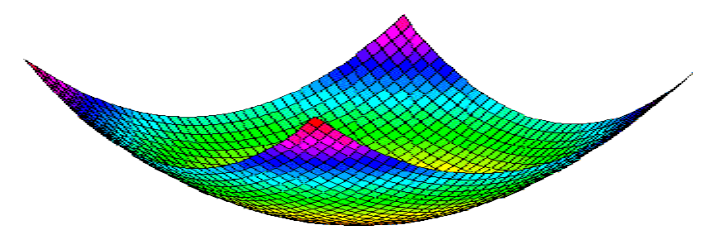

a) Networks assembled to form membrane

Membrane can be formed by many layers of networks piling up one on top of another; one network can also form a membrane by one way or another.

For example,

i) It firstly forms helical surface, and then a membrane can be formed by "compressing” the helical surface. 


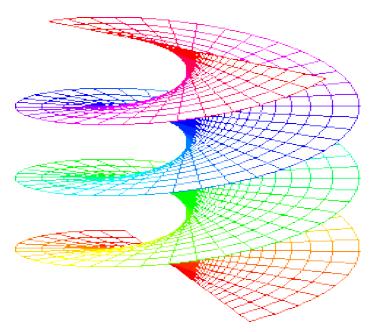

ii) An enneper can firstly be formed by a network, and then a membrane can also be formed by "compressing" the enneper.

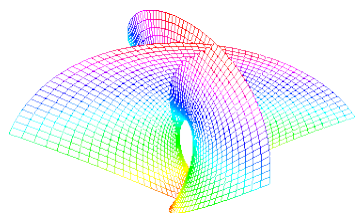

(Enneper)

Biomembrane is formed once the meshes of membrane become denser and smaller to some degree and the groups in the membrane control the opening and closing of the mesh. Cells can be formed on the basis of biomembrane. Cells are the real life unit, and they create life. Cell membrane is the most versatile of all the biomembrane which should be the best of all membrane formation; for example, cell membrane could easily transport the material along the direction of helix; Enneper can adjust the size of the holes of the cell membrane, thus selectively blocking or letting through certain ions. Details on the cell membrane will be discussed in further research.

b) Network assembled to form a cylinder

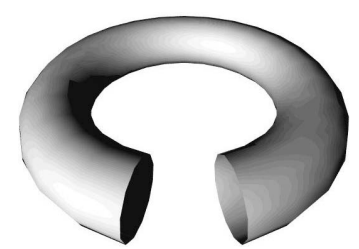

i) Two bending sections of cylinder could "bond" to become a ring surface.

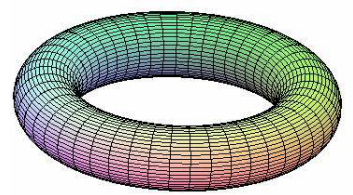

ii) A cylinder can form a trefoil knot when the length of the biomembrane is sufficient to form a cylinder.

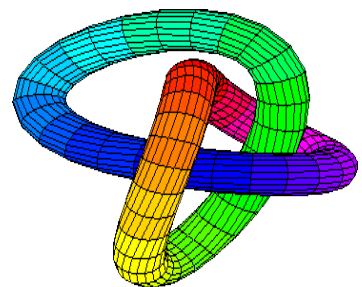

It is worth noting that all chains, belts or cylinders can form trefoil knots, so it is of high probability that the living matter is formed from trefoil knot. In fact, researchers found the trefoil knot virus in 2006 [4]. D. M. 
Wolba (University of Colorado) synthesized the Mobiusmolecule in 1982 [5]. Do other special living molecules exist? What kind of special properties do they have? All these questions are proposed for further explore and research.

\section{Discussion}

In the structure and function, there are essential differences between biomolecules and chemical molecules. The dual-branch loop helix (II) is the transition state from chemical molecules to biomolecules. After leaping over the transition state, the rigid structure of the molecule evolves into the topology structure. Meanwhile, in this system most of the dispersive, disordered thermodynamic energy is optimized to dynamic energy. The entropy in the system is translated from the probability entropy to the comentropy [6] [7], and the thermodynamic system evolves into dynamic system which no longer rigidly adheres to the principle of the thermodynamic theory. However, according to "theory of minimum rate of energy dissipation", a series of automatic control functions including self-reproduction, mass transfer, energy transfer, information transfer and so on is needed to complete the topology effect.

The evolution process from chemical molecules to biomolecules is a process with decreasing entropy. I. Prigogine [8] indicated evolution of entropy flow direction - the system absorbed the comentropy from the environment, but they neglected to see that the system entropy and energy quality had changed. We consider that the chemical substance is able to spontaneously evolve into living matter, not only because the system entropy decreases, but also the entropy and the energy quality have changed, in which the latter is more important. In fact, only by means of the reduction of system entropy is not sufficient to make chemical matter growing "function", for example, the perfect crystal has zero entropy (the Third Law of Thermodynamics), but it has no life functions. A series of changes from chain small molecules to the double helix through rotaxane, catenane and knot reflect the change of entropy and energy quality. The helix, especially the double helix having the highly ordered structure and full flexibility may implement all kinds of topologies. As a result, the information is stored and transmitted, system energy is integrated into controllable energy, and it can serve as a template for replication of various helical molecules. This should be the reason for nature selecting helix as the basis structure of the biomolecule.

\section{References}

[1] Jiang, Z. (2001) H Self-Organization Theory. Shanghai Scientific and Technological Literature Publishing House.

[2] Li, Q. and Hua, S. (1999) A Note on a Class of 3-Manifolds. Journal of Henan University (Natural Science), 2, 11-14.

[3] Van, G. and Lick, N. (1993) Theoretical Aspects of the Linked Ring Problem. New Journal of Chemistry, 17, 10-11.

[4] Olivier, G. (2006) Computational Biology. Public Library of Science, 15.

[5] Paul, H. (1997) Archimedes' Revenge (Chapter 6). China Translation \& Publishing Corporation.

[6] Yang, G. (1999) Information Entropy and Entropy. Scientific Chinese, 4, 51-52.

[7] Xing, X. (2001) Physical Entropy, Information Entropy and Its Evolution Equation. Science in China (Series A), 1, 7784.

[8] Iiya, P. and Isabelle, S. (2005) Order out of Chaos: The Men’s New Dialogue with Nature. Shanghai Translation Press. 